\title{
Quasielastic neutron scattering characterization of the relaxation processes in a room temperature ionic liquid
}

\author{
Alessandro Triolo ${ }^{\text {a) }}$ \\ Istituto per i Processi Chimico-Fisici-CNR, via La Farina 237, 98123 Messina, Italy \\ Olga Russina \\ Hahn-Meitner Institut, Glienicker Str. 100, D-14109 Berlin, Germany \\ Valeria Arrighi \\ Department of Chemistry, Heriot-Watt University, EH14 4AS, Edinburgh, United Kingdom \\ Fanni Juranyi \\ Laboratory for Neutron Scattering, PSI and ETH Zürich, CH-5232 Villigen-PSI, Switzerland \\ and Universität des Saarlandes, Im Stadtwald, D-66123 Saarbrücken, Germany \\ Stefan Janssen \\ Laboratory for Neutron Scattering, PSI and ETH Zürich, CH-5232 Villigen-PSI, Switzerland \\ Charles M. Gordon ${ }^{\text {b) }}$ \\ RWTH Aachen Institut für Technische Chemie und Makromolekular Chemie, Worringerweg 1, \\ 52066 Aachen, Germany
}

(Received 18 June 2003; accepted 4 August 2003)

\begin{abstract}
We report the first quasielastic neutron scattering measurements on a room temperature ionic liquid: 1- $n$-butyl-3-methylimidazolium hexafluorophosphate, $[\mathrm{bmim}]\left[\mathrm{PF}_{6}\right]$. Data were collected using a medium resolution spectrometer as a function of temperature in the range $250-320 \mathrm{~K}$. The data unequivocally indicate the existence of two different relaxation processes: a fast, localized motion occurring in the subpicosecond range and a slower process spanning the subnanosecond regime. These results provide experimental support to recently published molecular dynamics simulations. Evidence for slower, unresolved dynamics (under the present experimental conditions) is also obtained. Both temperature and momentum transfer dependence of the intermediate incoherent dynamic structure factor were investigated, after Fourier transformation into the temporal domain. The fast process shows no appreciable $Q$ - and $T$-dependence. On the other hand the slow process shows evidence of a complex, non-Debye and non-Arrhenius behavior. (C) 2003 American Institute of Physics. [DOI: 10.1063/1.1613637]
\end{abstract}

\section{INTRODUCTION}

Room temperature ionic liquids (RTILs) are a new class of chemicals that has recently attracted the attention of both academia and industry. They possess a number of extremely useful properties, which make them ideal solvents for a wide range of chemical reactions. RTILs consist of a bulky organic cation and an organic or inorganic anion. The nature of both cation and anion can strongly affect the chemical and physical properties of the corresponding salts. RTILs are good solvents for many organic and inorganic substances, the solubility properties being controlled by the structure of the salt. This allows one to bring "unusual combinations of reagents into the same phase." 1 They are nonvolatile and nonflammable. Although they may generally be regarded as polar solvents ${ }^{2}$ careful choice of the anion allows the formation of noncoordinating solvents. ${ }^{2(a)}$ Depending on the composition, they may be immiscible with selected organic solvents

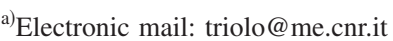

b)Electronic mail: gordon@itmc.rwth-aachen.de
}

(e.g., alkanes, alkenes, ethers), thus leading to biphasic systems which are ideally suited to catalytic systems or separations.

These properties are ideal for green chemical synthesis, as product separation is also facilitated by their low vapor pressure. Some of these materials are also immiscible with water, thus further facilitating extractions when dealing with nonvolatile products. Volatile products that are thermally stable may be removed simply by distillation from the nonvolatile RTIL. As a result, RTILs have been applied to a wide range of catalytic processes, especially those employing transition metal based and Lewis acid catalysis. ${ }^{3}$ The use of RTILs as solvents for biocatalysis is also developing. ${ }^{4} \mathrm{~A}$ number of reports indicate the enhanced capability of RTILs to host and enhance processes involving biomolecules, thus opening new avenues for nonaqueous enzymology.

These salts generally display low melting points combined with a high level of thermal stability. In addition, they display a large electrochemical window. In some cases the cation has even been involved in enhancing the efficiency of catalysis by direct interaction with a metal center. ${ }^{5}$

This enormous range of applications of RTILs as "de- 
signer solvents" requires a deeper understanding of their physical chemical properties in order to rationalize the correlation between structure and functionality.

The properties of RTILs have been previously investigated using a number of complementary techniques. Extensive thermodynamic characterization was carried out by Brennecke and $\mathrm{Gu}^{6}{ }^{6}$ who explored the volume expansivities and isothermal compressibilities of imidazolium and pyridinium-based RTILs. A detailed study of viscosity and conductivity in RTILs has recently appeared; ${ }^{7}$ in the same report, the authors demonstrated the existence of an unexpectedly large range of liquid fragilities for this class of materials. ${ }^{7}$ From a structural point of view, neutron diffraction data have recently been reported for $[\mathrm{mmim}] \mathrm{Cl}$ (Ref. 8) in the liquid phase. ${ }^{9}$ These measurements have highlighted the considerable degree of order existing in the liquid phase, closely resembling that found in the crystalline phase. This led to the conclusion that "in the liquid phase some short range associative structural ordering is retained." 9

A number of studies that provide information regarding the dynamic processes occurring in these materials have appeared in the literature. For example ultrafast solvent dynamics have been characterized by time-resolved optical Kerreffect spectroscopy. ${ }^{10}$ NMR spectroscopy has been used to determine the diffusion coefficients in a 1-ethyl-3methylimidazolium salt $[\mathrm{emim}]\left[\mathrm{BF}_{4}\right],{ }^{11}$ providing indication of a transition from discrete ion-pairs to individual ions. Finally $\mathrm{THz}$ dielectric spectroscopy has been applied to investigate the fast relaxations occurring in $[\mathrm{emim}][\mathrm{OTf}],{ }^{12}$ leading to the characterization of three Debye-type relaxations occurring in the subnanosecond regime. A number of molecular dynamics investigations have also appeared, providing detailed structural insight on RTILs. ${ }^{13}$ Dynamic studies are, however, still limited to the determination of an average value of the diffusion coefficient.

In this paper we report, for the first time, quasielastic neutron scattering (QENS) data on the fast relaxation dynamics in 1- $n$-butyl-3-methylimidazolium hexafluorophosphate, $[$ bmim $]\left[\mathrm{PF}_{6}\right]$. This technique allows us to obtain information on sub-nanosecond dynamics on a spatial scale of the order of a few $\AA$. QENS has been used in the past to investigate the dynamic properties of glassformers including polymeric materials, small molecules and amorphous salts. The technique makes it possible to extract the single-particle dynamics of the hydrogen atoms on a subnanosecond timescale which is not accessible using other techniques. Thus QENS is a unique tool in providing information which is complementary to that obtained using other experimental methods. Furthermore, by covering a spatial and temporal scale similar to that accessed by molecular dynamics simulations, QENS allows critical testing of the MD calculations.

Dynamic studies have been carried out by means of QENS on similar systems. For example, the single particle relaxation dynamics in the crystalline and liquid phases of $\left(n-\mathrm{C}_{10} \mathrm{H}_{21} \mathrm{NH}_{3}\right)_{2} \mathrm{MnCl}_{4},{ }^{14}$ has been probed between 1 and $600 \mathrm{ps}$ in the temperature range between 240 and $370 \mathrm{~K}$. This study provides insight into the nature of the transition from the highly regular, crystalline ordering of the alkyl chain to the amorphous liquid configurations. The simulta-

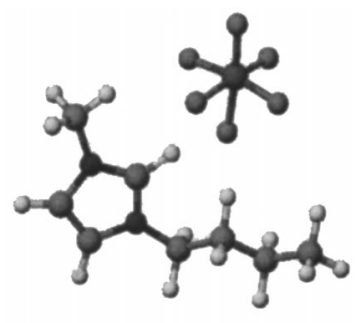

FIG. 1. (a) Schematic representation of the chemical structure of 1-n-butyl3-methylimidazolium hexafluorophosphate, $[\mathrm{bmim}]\left[\mathrm{PF}_{6}\right]$.

neous occurrence of thermally activated motions and cooperative torsion processes induces the melting of the alkyl chains. Investigations of the single particle dynamics of short alkyl chains have also been reported, such as QENS studies of the crystalline, plastic and liquid phases of butane $e^{15}$ as well as MD simulations of liquid butane. ${ }^{16}$

In this report, QENS data collected on a medium resolution spectrometer will be reported for $[\mathrm{bmim}]\left[\mathrm{PF}_{6}\right]$, providing the first experimental indication for the existence of two different kinds of relaxation dynamics in this material: a fast, confined motion inside a potential basin and a much slower relaxation showing distinctly non-Arrhenius behavior. The features of the latter process strongly resemble that of glass transition, including a substantial broadening of the relaxation shape.

\section{EXPERIMENT}

The synthesis of $[\mathrm{bmim}]\left[\mathrm{PF}_{6}\right]$ has been described previously. ${ }^{17}$ In order to ensure that the ionic liquid used was as pure as possible, all of the organic reagents were distilled prior to use. After preparation, the $[\mathrm{bmim}]\left[\mathrm{PF}_{6}\right]$ product was stirred with activated charcoal and acidic alumina at $50{ }^{\circ} \mathrm{C}$ for $24 \mathrm{~h}$. The charcoal and alumina were then removed by filtration of the neat liquid through celite. This procedure removes traces of coloured impurity. ${ }^{18}$ The structure of this salt is shown in Fig. 1. The glass transition $\left(T_{g}\right)$ of [bmim $]\left[\mathrm{PF}_{6}\right]$ is reported to occur at $\approx 212 \mathrm{~K} .{ }^{19}$ The value of $T_{g}$ is clearly strongly affected by the presence of even minor quantities of impurities such as moisture.

We observed that on heating an amorphous sample of [bmim $]\left[\mathrm{PF}_{6}\right]$ from low temperature (e.g., $T=100 \mathrm{~K}$ ) up to room temperature, crystallization occurs just above $T_{g}$. This crystalline phase melts at $\approx 280 \mathrm{~K}$. We collected neutron diffraction data (vide infra) for [ bmim $]\left[\mathrm{PF}_{6}\right]$ at $200 \mathrm{~K}$, both in the crystalline phase and in the supercooled amorphous phase. These data are reported in Fig. 2 and compared with recently published $x$-ray diffraction data collected on a similar material (namely $[\mathrm{bmim}] \mathrm{Cl}$ ) ${ }^{20}$ In the case of crystalline $[$ bmim $]\left[\mathrm{PF}_{6}\right]$, Bragg peaks are observed, which are not present in the supercooled sample. Many of the observed peaks correspond to those observed in the x-ray pattern of $[\mathrm{bmim}] \mathrm{Cl}$, indicating that the two types of radiations (x-ray and neutron) are probing similar structures. Differences in the x-ray and neutron patterns can be associated either to differences in the structure (the x-ray patterns refer to a sample with the $\mathrm{Cl}^{-}$anion, rather than the $\left[\mathrm{PF}_{6}\right]^{-}$) or to the fact that $\mathrm{x}$ rays probe the electron density map, while neu- 


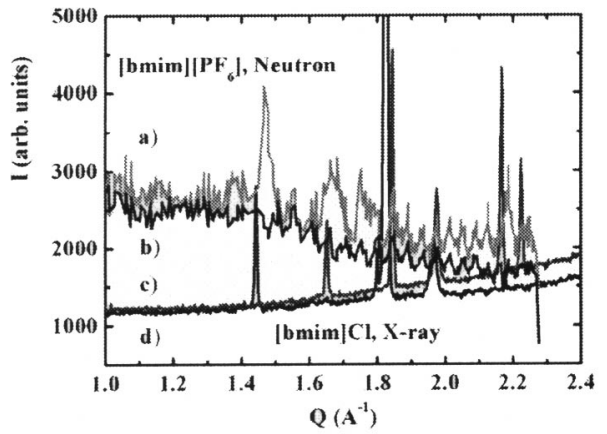

FIG. 2. Diffraction data from imidazolium-based RTILs: (a) Neutron diffraction data from crystalline $[\mathrm{bmim}]\left[\mathrm{PF}_{6}\right]$ at $200 \mathrm{~K}$; (b) neutron diffraction data from supercooled amorphous $[\mathrm{bmim}]\left[\mathrm{PF}_{6}\right]$ at $200 \mathrm{~K}$; (c) x-ray diffraction data from crystalline $[\mathrm{bmim}] \mathrm{Cl}$ (phase I) (Ref. 20); (d) x-ray diffraction data from crystalline [ $\mathrm{bmim}] \mathrm{Cl}$ (phase II) (Ref. 20).

trons probe the scattering length density map. At present we cannot provide structural information on this crystalline phase due to the limited angular range accessible and to the high noise of the data. We stress that these data have been collected on an instrument that is designed for dynamic characterizations and not for diffraction experiments (vide infra), as a consequence the $Q$ resolution of the neutron data is definitively poorer than the x-ray one; further measurements on adequate instruments are now planned to gain a better insight into the structural features of crystalline $[$ bmim $]\left[\mathrm{PF}_{6}\right]$. Due to the complex nature of $[\mathrm{bmim}]\left[\mathrm{PF}_{6}\right]$ phase diagram our study was limited either to temperatures above $280 \mathrm{~K}$ or, when below $280 \mathrm{~K}$, to supercooled systems. In the latter case, care was taken to study samples below 280 $\mathrm{K}$ after a sufficiently fast quenching from melt to the final temperature, so that no indications of the existence of the crystalline phase were observed. In particular, we ensured that no Bragg peaks were present in the neutron diffraction patterns that were collected. Under these conditions, relatively long measurement times $(\approx 3 \mathrm{~h}$ each) could be achieved with no appreciable indication of crystallization.

Before the QENS measurements, the sample was dried under high vacuum at $70{ }^{\circ} \mathrm{C}$ for $\approx 12 \mathrm{~h}$ to eliminate moisture. This procedure has been found to give liquids that are "operationally dry" (typical moisture content measured by Karl-Fisher titration $\left.=6 \times 10^{-3} \mathrm{M}\right) .^{21}$ After this treatment, the sample was poured into a flat cell with a sample thickness of $0.2 \mathrm{~mm}$, a condition that ensures minimization of multiple scattering effects while maintaining a reasonable scattering intensity. Typical acquisition times were about $3 \mathrm{~h}$.

QENS measurements were carried out at the FOCUS instrument at the SINQ installation. ${ }^{22}$ This is a hybrid timeof-flight spectrometer combining a doubly focusing crystal monochromator (variable curvatures in both directions) with a fast rotating Fermi-chopper. In this case, the instrument was used in the time focusing mode. At the chosen wavelength $(\lambda=5.0 \AA)$, using the PG002 monochromator reflection, we accessed energy exchanges between -2 and +20 $\mathrm{meV}$ neutron energy loss and gain, respectively. A resolution of $90 \mu \mathrm{eV}$ (FWHM) was achieved. Detectors were located at different scattering angles covering the range $10^{\circ}<2 \theta$ $<130^{\circ}$. Data reduction was performed using the IDA software (http://www.e13.physik.tu-muenchen.de/Wuttke/ Ida.html) made available to the authors by Dr. A. Meyer (Technische Universität München, Germany). The software allowed correction for sample absorption and transmission as well as subtracting the empty cell contribution. The QENS data were then normalized to the vanadium scattering. This procedure leads to the incoherent dynamic structure factor at constant scattering angle. Reduction to constant momentum transfer has been obtained by interpolating the energy-angle data into the energy- $Q$ space using the IDA software; the corresponding data were defined in the $Q$ range included between 1.4 and $2.2 \AA^{-1}$.

After the conventional data reduction described above, the $S(Q, \omega)$ data were transformed from the energy domain into the temporal one by means of Fourier transformation (FT). This operation was done using the FURY software available at ISIS (RAL, UK). ${ }^{23}$ The outcome of this procedure is the Intermediate Incoherent Dynamic Structure Factor, $I(Q, t)$. This function is obtained after normalization of the FT of the experimental data by the corresponding function obtained from the instrumental resolution, measured with a vanadium slab at the same orientation as the sample. In this way, $I(Q, t)$ was defined for $4.1 \times 10^{-4}<t(\mathrm{~ns})<2.5$ $\times 10^{-2}$. The advantages of using data defined in the time domain are various: the physical meaning of $I(Q, t)$ is much more intuitive than the one of the corresponding function in the energy domain. Also the effect of the instrumental resolution is eliminated by the use of the above mentioned normalization, thus making comparison of different data sets more straightforward.

The spectrometer was also used to collect diffraction data, averaging over the entire time of flight data set. Data collected on the amorphous samples did not show any appreciable feature, as the scattering is dominated by the incoherent contribution of $\mathrm{H}$ atoms. In the case of crystalline samples (see Fig. 2), Bragg peaks associated with the lattice geometry could be detected.

In this paper, we report on QENS data for $[\mathrm{bmim}]\left[\mathrm{PF}_{6}\right]$, at $T=250,260,270,280,290,300,310$, and $320 \mathrm{~K}$.

We also report data collected on the backscattering instrument IN16 at the ILL (FR). ${ }^{24}$ We will discuss the IN16 data in greater detail in a forthcoming paper. At present we limit ourselves to the presentation of the fixed energy scans collected on this instrument. IN16 has been used in order to access slower dynamics than that probed by FOCUS. In particular, the chosen wavelength was $6.271 \AA$, allowing a resolution of $0.9 \mu \mathrm{eV}$ to be obtained. The accessible $Q$ range spans from 0.4 to $1.9 \AA^{-1}$. Data collection involved monitoring of the number of neutrons scattered elastically during heating (or cooling) of the sample. At very low temperatures no dynamic processes are active, and no energy exchange with neutrons can occur. As soon as the temperature increases sufficiently to activate some relaxation, this process can exchange energy with neutrons, whose energy will then change. The consequence of this interaction is that a drop in the intensity of the elastically scattered neutrons will be observed. Such an approach turns out to be extremely useful when dealing with relaxation of sidegroups ${ }^{25}$ or with com- 

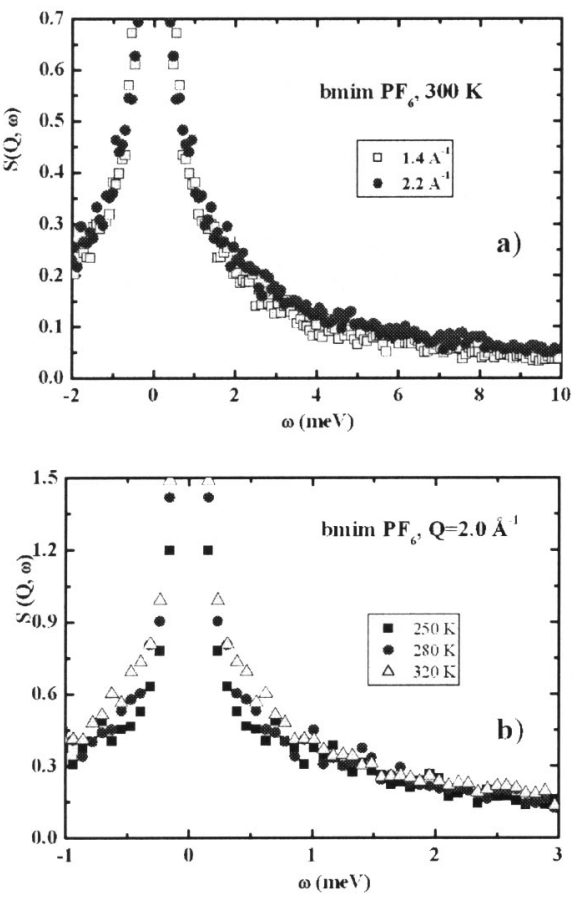

FIG. 3. (a) QENS data from $[\mathrm{bmim}]\left[\mathrm{PF}_{6}\right]$ at $T=300 \mathrm{~K}$ and two selected values of momentum transfer $\left(Q=1.4\right.$ and $\left.2.2 \AA^{-1}\right)$; (b) QENS data from [bmim $]\left[\mathrm{PF}_{6}\right]$ at $Q=2.0 \AA^{-1}$ and three selected temperatures ( $T=250,280$, and $320 \mathrm{~K})$.

plex (bio)-macromolecules. ${ }^{26}$ For this experiment we used a cylindrical geometry, with a sample thickness of $0.15 \mathrm{~mm}$ and a sample diameter of $20 \mathrm{~mm}$. We explored the temperature range between 12 and $400 \mathrm{~K}$.

\section{RESULTS}

A representative set of QENS data for $[$ bmim $]\left[\mathrm{PF}_{6}\right]$ is reported in Fig. 3. In Fig. 3(a), the $Q$ dependence of the dynamic incoherent structure factor is shown at a fixed temperature $(300 \mathrm{~K})$, while in Fig. 3(b) data collected at different temperatures are reported for a fixed value of the momentum transfer $Q\left(Q=2.0 \AA^{-1}\right)$.

The data in Figs. 3(a) and 3(b) give qualitative information on the dynamic behavior of $[\mathrm{bmim}]\left[\mathrm{PF}_{6}\right]$. Close inspection indicates that: (i) there is a strong elastic signal (i.e., the narrow central peak) due to the existence of slow dynamic processes which are not resolved under the present experimental conditions (chosen instrumental resolution and selected $Q$ and temperature ranges); and (ii) in addition to elastic scattering, even at the lowest temperature probed (250 $\mathrm{K})$ a broad quasielastic component is observed. This indicates that, although under the present experimental conditions it is not possible to follow the complete relaxation, dynamic processes do occur within the accessible energy window at these temperatures. The quasielastic component is both temperature and $Q$ dependent: molecular motion becomes faster [as indicated by the progressive broadening of the $S(Q, \omega)$ data] with either increasing temperature or momentum transfer. As will be described in detail later, careful inspection of the QENS data indicates that the quasielastic broadening consists of two different contributions: a faster component with full width at half maximum (FWHM) of $\approx 4$ $\mathrm{meV}$ and a slower process with a FWHM of $\approx 0.5-1 \mathrm{meV}$.

In order to analyze the QENS data quantitatively, we decided to perform a Fourier transformation from the $Q-\omega$ space to the $Q-t$ space. This procedure, after normalization with the corresponding contribution from the instrumental resolution, yields $I(Q, t)$. This function is a measure of the loss of self-correlation of the local density of $\mathrm{H}$ atoms, $\rho_{\mathrm{H}}(r, t)$. In the case of a simple relaxation process, the shape of $I(Q, t)$ is described by a simple exponential decay: $I(Q, t) \propto e^{-t / \tau}, \tau$ being the characteristic time for the relaxation process. However, deviations from this behavior are often observed in the relaxation dynamics of glassforming systems and the relaxation processes are described using nonexponential functions such as the stretched exponential: $I(Q, t) \propto \exp [-t / \tau]^{\beta}$, where $\beta$ is a stretching parameter accounting for the deviations from the simple exponential law. ${ }^{27}$

It has been proposed that the $\beta$ parameter might be related to the degree of cooperativity of the relaxation process, ${ }^{28}$ which implies a homogeneous nonexponential shape for the relaxation. Alternatively it has been suggested that the nonexponential behavior may be due to the superposition of simple exponential relaxations, leading to a process that is characterized by a distribution of relaxation times. ${ }^{29}$ Whatever the explanation for this complex behavior, the use of the stretched exponential [often indicated as KohlrauschWilliams-Watts (KWW) function] is generally limited to data that are defined in the temporal domain. Moreover, analysis of QENS data in the frequency domain requires convolution of the model function (in this case the FT of the KWW) with the experimental resolution, thus making fitting a complex computational task. On the other hand, when dealing with QENS data after Fourier transformation into the time domain, the effect of the resolution can easily be accounted for and this simplifies subsequent data analysis.

For these reasons, we decided to transform our QENS data for $[\mathrm{bmim}]\left[\mathrm{PF}_{6}\right]$ into the time domain, thus obtaining the corresponding $I(Q, t)$ data. In Fig. 4(a), we report the temperature dependence of the $I(Q, t)$ for $[\mathrm{bmim}]\left[\mathrm{PF}_{6}\right]$ at a fixed value of $Q$. The bimodal nature of the dynamic process is now evident with two relaxations occurring over the experimental time range. The faster event occurs on a time scale of the order of fractions of picoseconds, and a much slower process involves relaxation over a time scale of $\approx 0.01 \mathrm{~ns}$. The time domain data support the qualitative observations made earlier for the dynamic incoherent structure factor. Furthermore, within the experimental time range, the relaxation process does not decay to zero even at the highest temperature investigated $(T=320 \mathrm{~K})$. This result is consistent with the existence of an elastic peak in the frequency domain data and indicates that measurements at higher temperatures and/or higher resolution are required to describe the whole relaxation process as slower dynamic events exist in the sample at these temperatures. A qualitative inspection of these data indicates that the dynamics are influenced by the temperature, becoming faster as the temperature increases. However, it appears that the slow process is more strongly affected by temperature than the faster one. Similar 

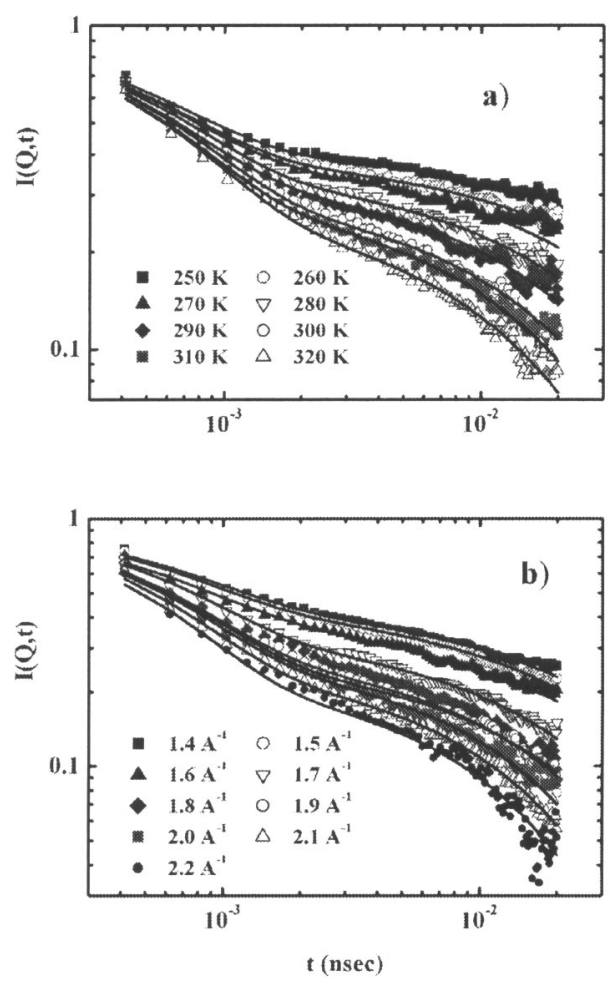

FIG. 4. (a) Temperature dependence of $I(Q, t)$ for $[\mathrm{bmim}]\left[\mathrm{PF}_{6}\right]$ at $Q$ $=2.0 \AA^{-1}$. The symbols refer to experimentally derived data, while the lines represent the fit in terms of the model outlined in the text. (b) Momentum transfer dependence of $I(Q, t)$ for $[\mathrm{bmim}]\left[\mathrm{PF}_{6}\right]$ at $T=320 \mathrm{~K}$. The symbols refer to experimentally derived data, while the lines represent the fit in terms of the model outlined in the text.

behavior can be observed for the $Q$ dependence of the $I(Q, t)$. In Fig. 4(b) we show the corresponding data at the selected temperature, $T=320 \mathrm{~K}$.

Following the qualitative analysis described above, a quantitative description of the experimental data can be achieved by accounting for the existence of two distinct processes. Thus the intermediate scattering function was fitted according to

$$
I(Q, t)=\left[A \exp \left(-t / \tau_{\beta}\right)+(1-A)\right] \exp \left(-t / \tau_{\alpha}\right)^{\beta},
$$

where $A$ is the amplitude of the faster process (hereafter referred to as the $\beta$-process), $\tau_{\beta}$ is its characteristic time, ( 1 $-A$ ) is the amplitude of the slower process (hereafter referred to as the $\alpha$-process) whose characteristic time is $\tau_{\alpha}$, and $\beta$ is the stretching parameter of the $\alpha$-process. The model reported in Eq. (1) accounts for two processes: the faster has a purely exponential shape, while the slower has a non-Debye shape, with the stretching parameter, $\beta$, accounting for deviations from purely exponential relaxation. ${ }^{27}$ No further slower component was needed to describe the decay of the $I(Q, t)$ data, as the $\alpha$-process accounts for the complete relaxation. Preliminary data analysis also led to the conclusion that the $\beta$-process is characterized by a stretching parameter $\beta \sim 1$, corresponding to the simple exponential relaxation shape used in Eq. (1). The continuous lines shown in Figs. 4(a) and 4(b) are the fits to the experimental data in terms of the model function given by Eq. (1). For each data set, the fitting parameters are $A, \tau_{\alpha}, \tau_{\beta}$, and $\beta$. In order to obtain a consistent scenario for the complete data sets, we imposed a number of conditions on these parameters. In particular, we assumed that the temperature dependence of the $\alpha$-process is given by

$$
\tau_{\alpha}(T)=\tau_{\alpha}^{0} \exp \left[E_{a} / k_{B}\left(T-T_{0}\right)\right],
$$

where $\tau_{\alpha}^{0}$ corresponds to the characteristic time at infinite temperature, $E_{a}$ is an apparent activation energy, $k_{B}$ is the Boltzmann constant, and $T_{0}$ is a characteristic temperature where the relaxation time diverges. Such an empirical law, often indicated as Vogel-Fulcher-Tammann equation, has been shown to provide an adequate description of the temperature dependence of the relaxation time in glassforming materials. In principle, a value of $T_{0}=0$ would imply Arrhenius-type behavior.

It emerges from a number of investigations ${ }^{30}$ on hydrogenous materials that the slow dynamics above $T_{g}$ are characterized by a power law dependence of the characteristic time versus $Q$ [see Eq. (3)]. Preliminarily fits were performed with $\tau_{\alpha}(Q)$ as an adjustable parameter to confirm that, within the experimentally accessible $Q$ range, a unique power law defined the $Q$-dependence of the characteristic time. These preliminary fits indicated that the $\tau_{\alpha}$ values could be expressed in terms of a power law dependence of the type,

$$
\tau_{\alpha}(Q)=\tau_{\alpha}^{0} Q^{-\nu},
$$

where $\nu$ is the exponent characterizing the $Q$ dependence and $\tau_{\alpha}^{0}$ corresponds to $\tau_{\alpha}\left(Q=1 \AA^{-1}\right)$. Equation (3) was then used to decrease the number of independent parameters involved in the fits, while still maintaining a physical meaning for the resulting parameters.

In using Eq. (1) to fit the experimental data, we allowed $\beta$ to vary both with temperature and $Q$ (accounting for nonDebye behavior of the $\alpha$-process). The KWW is an empirical function often used to represent the experimental data. Values of $\beta<1$ often indicate the existence of a distribution of relaxation times although it has been shown that $\beta<1$ may also be associated with a bimodal relaxation, ${ }^{31}$ where two different relaxation processes concur in providing loss of correlation.

Due to the chemical complexity of the system investigated here, it might be expected that the decay of the $I(Q, t)$ data results from a superposition of different relaxation processes, each characterized by its own $T$ and $Q$ dependence. If this is the case, the $Q / T$ dependence of relaxations may show a complex behavior stemming from this superposition: the $\beta$ parameter resulting from the fits would then show some $Q / T$ dependence, as a single relaxation function is used to account for a superposition of processes in a phenomenological way.

In Fig. 5, the relaxation map at a selected $Q$ value $(Q$ $=2.0 \AA^{-1}$ ) obtained for $[$ bmim $]\left[\mathrm{PF}_{6}\right]$ from the fit of the data with the model of Eq. (1) is reported. In particular, the average characteristic times for both the fast $\beta$ - and slow $\alpha$-processes are given as a function of $1000 / T$ [note that $\left\langle\tau_{\alpha}\right\rangle=\tau_{\alpha} / \beta \Gamma(1 / \beta)$ is reported for the $\alpha$-process, $\Gamma$ being the Gamma function]. The characteristic time $\tau_{\beta}$ is essentially temperature independent, while highly non-Arrhenius behavior of the $\alpha$-process can be observed. In the inset, the 


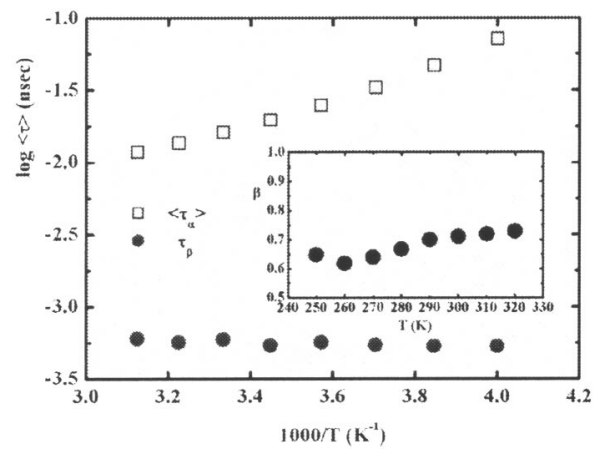

FIG. 5. Temperature dependence of the characteristic times of the fast $\left(\tau_{\beta}\right)$ and slow $\left(\left\langle\tau_{\alpha}\right\rangle\right)$ relaxations for $Q=2.0 \AA^{-1}$. In the inset the temperature dependence of the stretching parameter $\beta$ is reported for the same $Q$ value.

temperature dependence of the stretching parameter of the $\alpha$-process, $\beta$, is reported for $Q=2.0 \AA^{-1}$ : in this case the $\beta$ parameter increases with increasing temperature.

Figure 4(b) contains a representative set of data recorded at fixed temperature as a function of the momentum transfer, $Q$. From a qualitative point of view, increasing $Q$ has an effect that is equivalent to that observed when increasing temperature. In Fig. 6, the momentum transfer dependence of the characteristic times for the two relaxation processes is reported as a function of $\log Q$. The negligible $Q$ dependence of $\tau_{\beta}$ can be noticed, while $\left\langle\tau_{\alpha}\right\rangle$ follows a power law behavior which was explicitly considered in the fitting procedure. We note that a linear $\log -\log$ dependence has been assumed for $\tau_{\alpha}$ and not for $\left\langle\tau_{\alpha}\right\rangle$ : this explains the slight deviations of $\left\langle\tau_{\alpha}\right\rangle$ from a linear trend that are due to the $Q$ dependence of the $\beta$ parameter. The latter is found to monotonously increase from $\approx 0.6$ (at $Q=1.4 \AA^{-1}$ ) to $\approx 0.8$ (at $Q$ $=2.2 \AA^{-1}$ ) as $Q$ increases (inset of Fig. 6).

\section{DISCUSSION}

The QENS data for $[$ bmim $]\left[\mathrm{PF}_{6}\right]$ reported here provide clear evidence for the existence of (at least) two dynamic processes in the region of room temperature. The time scale of these processes falls in the subpicosecond and subnanosecond regimes and their divergence increases as the tem-

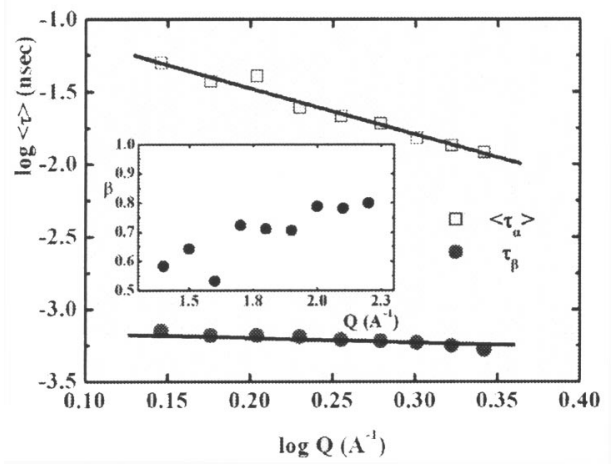

FIG. 6. Momentum transfer dependence of the characteristic times of the fast $\left(\tau_{\beta}\right)$ and slow $\left(\left\langle\tau_{\alpha}\right\rangle\right)$ relaxations for $T=300 \mathrm{~K}$. In the inset the $Q$ dependence of the stretching parameter $\beta$ is reported for the same temperature. perature decreases. QENS data contain information on the single particle dynamics over a microscopic region $(r$ $\sim 3 \AA$ ). Over this distance scale, the loss of self-correlation has been shown to occur through two main mechanisms: the fast $\beta$ and the much slower (more than one decade in time) $\alpha$-process. This observation supports recent results from molecular dynamics simulations carried out on the same material at $300 \mathrm{~K}$, where analysis of the mean-square displacement of the $[\mathrm{bmim}]^{+}$unit indicated the existence of two processes characterized by different time scales. ${ }^{13}$ The time scales associated with these motions fall in the same range in which we identify the $\alpha$ - and $\beta$-processes. A direct comparison between our experimental results and simulations is in progress in order to account for the details of the microscopic dynamics of these molecules.

The two dynamic processes show notably different behavior. In particular, the characteristic relaxation time for the fast $\beta$-process shows negligible $Q$ and $T$ dependence (see Figs. 5 and 6). The existence of such a dynamic component occurring in the subpicosecond regime has been reported for a number of glassforming materials, from polymers to small molecules to ionic mixtures. We thus surmise that the fast process results from highly localized librational motions, associated with the $\mathrm{H}$ atoms visiting local energy minima (basins) in the phase space. For such motion, negligible $T$ and $Q$ dependence is expected. The quantity $(1-A)$ in Eq. (1) can be considered as a Debye-Waller factor arising from the basin confinement. Its $Q$ dependence should give information on the spatial extent of the confinement. We found that $(1-A)$ follows the relationship $\exp \left[-\left\langle z^{2}\right\rangle Q^{2} / 3\right]$, with $z$ $\sim 0.7 \AA$ being the average size of the basin in which the librational motion occurs during the fast $\beta$-process. This information allows the characterization of a relaxation event over a temporal window of a picosecond, representing a channel of significant self-correlation loss. For example at $Q=2.0 \AA^{-1}$, the drop in self-correlation associated to this process is between $60 \%$ and $70 \%$, depending on the temperature.

The residual self-correlation finds a further channel of loss through the $\alpha$-process. Despite the fact that our data do not allow observation of a complete loss of correlation through this process, as indicated also by the existence of the elastic signal in the $S(Q, \omega)$ data (see Figs. 3 and 4), we do not find indications that more than two processes are involved in the overall relaxation. In Eq. (1) we do not need to introduce a flat background associated with a saturation of the loss due to the $\alpha$-process, which might appear as an intermediate plateau, a precursor for a further slower dynamic process. On the other hand the strongly non-Debye nature of the $\alpha$-process (as indicated by values for the $\beta$ parameter being systematically lower than 1) might be an indication either of a complex single relaxation process, or of a superposition of dynamic processes, or even a combination of these effects. The present data set does not allow conclusive statements on this issue, which require a more detailed and complete data set. However some preliminary information can still be derived at the present stage.

There is a variety of possible dynamic events which can occur in amorphous $[$ bmim $]\left[\mathrm{PF}_{6}\right]$. The methyl and the butyl 


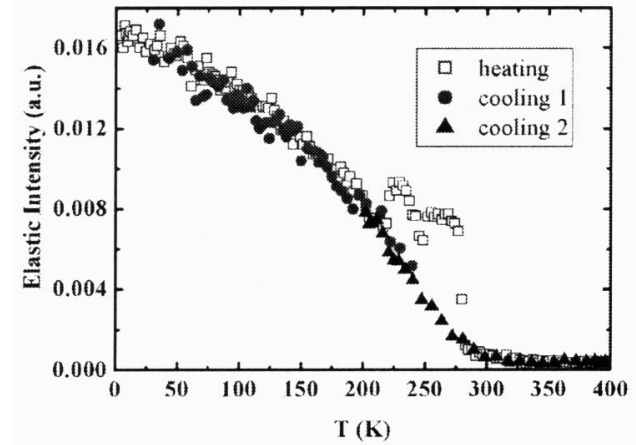

FIG. 7. Fixed energy scan of $[$ bmim $]\left[\mathrm{PF}_{6}\right]$ at $Q=1.9 \AA^{-1}$, as collected on IN16. The data refer to two cooling and one heating cycle. During the heating cycle crystallization occurs above $T_{g}$. When cooling from the melt phase, supercooling occurs and the sample remains amorphous.

substituents are relatively mobile units. The data collected on IN16 allow us to observe that these units start to relax at temperatures as low as $\approx 100 \mathrm{~K}$. A large number of investigations ${ }^{25,32}$ on methyl groups belonging to glassy systems indicates that a characteristic hopping process occurs starting at $\approx 100 \mathrm{~K}$, where the $\mathrm{CH}_{3}$ unit (directly attached to the $\mathrm{N}$ atom of the imidazolium ring) can rotate with a threefold symmetry about the $\mathrm{N}-\mathrm{C}$ axis. At comparable instrumental resolution, recent backscattering data from one of us showed that butyl chains directly connected to a glassy polymer skeleton also trigger their dynamics at $\approx 100 \mathrm{~K}^{33} \mathrm{Ac}$ cordingly both alkyl side groups in $[\mathrm{bmim}]\left[\mathrm{PF}_{6}\right]$ would be expected to show relaxational dynamics from $100 \mathrm{~K}$.

Figure 7 shows data from $[\mathrm{bmim}]\left[\mathrm{PF}_{6}\right]$ collected on IN16 (ILL) at a selected value of the momentum transfer. This shows data from three different thermal paths. The sample was first cooled from room temperature to $10 \mathrm{~K}$ ("cooling 1"). This step was followed by heating to $400 \mathrm{~K}$ ("heating"). In order to check the feasibility of supercooling the sample we further cooled it from 400 to $200 \mathrm{~K}$ ("cooling 2 "). It appears that once an amorphous sample is obtained, it may be easily supercooled. On the other hand upon heating the sample from $T<T_{g}$ it invariably crystallized as soon as $T_{g}$ was reached. The combination of methyl and butyl group relaxation induces a loss of elastic signal beginning at $\approx 100$ $\mathrm{K}$ [we note that the decrease in signal in the range 10 $<T(\mathrm{~K})<100$ is linear with temperature and corresponds to the Lamb-Mossbauer effect]. ${ }^{25}$ Such behavior does not convey relevant information for the present characterization. At present we are not able to separate the contribution from the two alkyl units. Further analysis of these data, and possibly on that from selectively deuterated samples should provide a clearer information on the sub- $T_{g}$ dynamics.

The resolution of the two instruments currently being employed is very different. IN16 allows the detection of motion that is approximately two decades slower than that accessible to FOCUS. However, we wish to stress that measurements on IN16 show that both methyl and butyl group motion are clearly active from $100 \mathrm{~K}$. Such complex dynamics will presumably play a role in determining the overall relaxation dynamics of $[\mathrm{bmim}]\left[\mathrm{PF}_{6}\right]$ at ambient temperatures. We expect that the process characterized from the
FOCUS data around room temperature and indicated as the $\alpha$-process might be related to the side group relaxations. It is expected that the $\mathrm{H}$ atoms directly linked to the heterocyclic ring will only show fast librational dynamics, presumably similar to those indicated for the $\beta$-process. At the chosen $Q$ values, translational diffusion should play a minor role and, consequently, we should be observing mainly conformational transitions of the two side chains.

In order to disentangle these two contributions more detailed measurements are required, preferably using selectively deuterated materials. At the present stage, we can only interpret the results in a phenomenological manner, without providing a detailed molecular interpretation of the dynamic processes involved. The slow $\alpha$-process has been found to display distinctly non-Debye and non-Arrhenius behavior (see Figs. 5 and 6). The residual self-correlation from the previous, faster $\beta$-process is further dissipated through a channel whose features are reminiscent of the cooperativity which characterizes the glass transition. It is well known that small molecules, when cooled towards their $T_{g}$, manifest an increasing trend towards cooperative relaxation events, which are distinguished by a progressive decrease of the stretching parameter. Moreover the closer to $T_{g}$, the longer the relaxation time with diverging behavior which is typically modeled in terms of the VTF law [Eq. (2)]. These features, which might be considered as fingerprints for the glass transition, can be found on the basis of the analysis of our present QENS data on $[\mathrm{bmim}]\left[\mathrm{PF}_{6}\right]$. As shown in the inset of Fig. 5, the stretching parameter decreases together with temperature. This behavior is common to many glassformers, including supercooled water ${ }^{34}$ and indicates that at high temperature, the $\alpha$-process loses its heterogeneous features and tends towards a simple Debye-type relaxation.

The temperature dependence of our QENS data could be well described in terms of a VTF law [Eq. (2)] with the following parameters: $E_{a}=2.4 \mathrm{~kJ} / \mathrm{mol} ; T_{0}=173 \mathrm{~K}$. Moreover $\tau_{\alpha}^{0}$ showed a $Q$ dependence which was described in terms of Eq. (3), with $\tau_{\alpha}^{0}\left(Q=1 \AA^{-1}\right)=7.2 \times 10^{-11} \mathrm{~s}$ and $\nu$ $\sim 2.5$. In the case of Gaussian dynamics, it has been shown that a connection should exist between the parameters $\beta$ [Eq. (1)] and $\nu$ [Eq. (3)]. In particular, one should find: $\nu_{\text {Gauss }}$ $=2 / \beta$. In our case, with $\beta \sim 0.7$, we derive $\nu_{\text {Gauss }} \sim 2.85$, in fairly good agreement with the experimental result. By comparison, the corresponding $\nu$ value from MD simulations for supercooled water in the same range of $Q$ was $2 .{ }^{34}$ Although this result must be considered with care due to the different molecular topology, similar values are also obtained from QENS data on polymers. ${ }^{30}$

These values for the VTF law are in agreement with recent Brillouin scattering results obtained in our laboratory ${ }^{35}$ while the temperature dependence of the static viscosity, ${ }^{36}$ though showing the same characteristic temperature $T_{0}$, has a higher apparent activation energy $E_{a}$. The latter finding might be rationalized when one considers that the viscosity involves cooperative relaxation events, notably mutual interactions between the different portions of the chains. Entanglements effects play a large role in determining the bulk viscosity which then can be expected to have a 
higher apparent activation energy than conformational transitions.

In our discussion of the experimental data, the observed relaxation processes have been directly related to motions associated to portions of the cation. No explicit mention has been made on the role that the anion, namely the $\left[\mathrm{PF}_{6}\right]^{-}$ anion may play on determining the observed features. This approach is justified by the consideration that the QENS technique mainly probes the $\mathrm{H}$ atom motion, thus the observed QENS patterns reflect the dynamics of the cation, probed by the $\mathrm{H}$ atom motions. On the other hand it is intuitive that the anion may play a role in determining the relaxation processes occurring in the material, as noted by a referee. In order to address this point, we carried out a fixed energy scan on IN16 on a sample of $[\mathrm{bmim}]\left[\mathrm{Tf}_{2} \mathrm{~N}\right]$ $\left(\left[\mathrm{Tf}_{2} \mathrm{~N}\right]=\left(\mathrm{CF}_{3} \mathrm{SO}_{2}\right)_{2} \mathrm{~N}\right.$, data not shown). Thus, the only difference from $[\mathrm{bmim}]\left[\mathrm{PF}_{6}\right]$ is in the nature of the anion. The two data sets are essentially the same, allowing for the difference in glass transition temperature (in particular, we have: $\quad T_{g}\left([\mathrm{bmim}]\left[\mathrm{Tf}_{2} \mathrm{~N}\right]\right)=187 \mathrm{~K}, \quad T_{g}\left([\mathrm{bmim}]\left[\mathrm{PF}_{6}\right]\right)$ $=212 \mathrm{~K}){ }^{\dagger}{ }^{\dagger}$ This result, which will be discussed in greater detail elsewhere, indicates that despite the large structural effect played by the anion in determining the degree of local ordering, the QENS technique does not detect differences in the relaxation processes when the anion is changed. If there is any difference this is covered by the macroscopic difference in glass transition.

\section{CONCLUSION}

In this paper we report the first example of QENS data from a room temperature ionic liquid, 1- $n$-butyl-3methylimidazolium hexafluorophosphate, $\left[\mathrm{bmim}^{\mathrm{m}}\right]\left[\mathrm{PF}_{6}\right]$, over the temperature range 250 to $320 \mathrm{~K}$ using a medium resolution spectrometer. These data are used to derive the intermediate incoherent dynamic structure factor, $I(Q, t)$, in the dynamic window: $4.1 \times 10^{-4}<t(\mathrm{~ns})<2.5 \times 10^{-2}$. The data indicate the existence of two different relaxation processes occurring in the sample over the probed time/ temperature ranges. First there is a faster $\beta$-process that shows no appreciable $Q$ and $T$ dependence. This has been interpreted in terms of motion inside a cage, whose spatial extent is approximately $0.7 \AA$. The $\mathrm{H}$ atoms (whose motion is probed by incoherent QENS) perform fast dynamics inside narrow energy basins of the phase space. After a sufficiently long time (fractions of nsec), conformational transitions become accessible. These slower dynamic processes are superimposed to give the overall experimentally accessible $I(Q, t)$. This complex relaxation (indicated as the $\alpha$-process) has unequivocally non-Debye behavior, with a stretching parameter $\beta$, which depends on both temperature and momentum transfer. The temperature dependence of this process is also non-Arrhenius in nature. This behavior has been modelled in terms of a VTF law [Eq. (2)], leading to an apparent activation energy, $E_{a}=2.4 \mathrm{~kJ} \mathrm{~mol}^{-1}$ and a characteristic temperature $T_{0}=173 \mathrm{~K}$. The $Q$-dependence of this process has also been investigated, leading to approximately Gaussian dynamics, as the relationship $\nu=2 / \beta$ was found to be followed approximately.
The present data unravel important new insights into the relaxational dynamics of a model RTIL. A quantitative comparison with results from MD simulations is presently in progress. Moreover new QENS measurements at complementary instrumental resolutions and temperatures are also underway, in order to further extend the available time/ temperature range and better describe the nature of the dynamic processes.

\section{ACKNOWLEDGMENTS}

This work was partially performed at the spallation neutron source SINQ, Paul Scherrer Institut, Villigen, Switzerland. We also thank ILL (Grenoble, FR) for providing us with the beam time on IN16, whose local contacts (B. Frick and T. Seydel) are gratefully acknowledged for their support during the measurements. A.T. is grateful to D. Arigo' and G. Spinella (IPCF-CNR) for their skillfull expertise in the QENS cell manufacturing. The authors thank Professor $\mathrm{H}$. Hamaguchi (Tokio University) for making the X-ray diffraction data reported in Fig. 2 available.

${ }^{1}$ T. Welton, Chem. Rev. (Washington, D.C.) 99, 2071 (1999).

${ }^{2}$ See, e.g. (a) M. J. Muldoon, C. M. Gordon, and I. R. Dunkin, J. Chem. Soc., Perkin Trans. 2 2, 433 (2001); (b) S. N. V. K. Aki, J. F. Brennecke, and A. Samanta, Chem. Commun. (Cambridge) 2001, 413; (c) S. N. Baker, G. A. Baker, M. A. Kane, and F. V. Bright, J. Phys. Chem. B 105, 9663 (2001)

${ }^{3}$ (a) J. Dupont, R. F. de Souza, and P. A. Z. Suarez, Chem. Rev. (Washington, D.C.) 102, 3667 (2002); (b) H. Olivier-Bourbigou and L. Magna, J. Mol. Catal. A: Chem. 182, 419 (2002); (c) C. M. Gordon, Appl. Catal., A 222, 101 (2001); (d) R. Sheldon, Chem. Commun. (Cambridge) 2001, 2399.

${ }^{4}$ See, e.g., P. Lozano, T. deDiego, D. Carrié, M. Vaultier, and J. L. Iborra, Chem. Commun. (Cambridge) 2002, 692.

5 (a) L. Xu, W. Chen, and J. Xiao, Organometallics 19, 1123 (2000); (b) C. J. Mathews, P. J. Smith, T. Welton, and A. J. P. White, ibid. 20, 3848 (2001).

${ }^{6}$ Z. Gu and J. F. Brennecke, J. Chem. Eng. Data 47, 339 (2002).

${ }^{7}$ W. Xu, E. I. Cooper, and C. A. Angell, J. Phys. Chem. B 107, 6170 (2003).

${ }^{8}$ In this paper, the nomenclature of cations based on the imidazolium cation is as follows: $\mathrm{mmim}=1,3$-dimethylimidazolium; emim=1-ethyl-3methylimidazolium; bmim=1-n-butyl-3-methylimidazolium

${ }^{9}$ C. Hardacre, J. D. Holbrey, S. E. J. McMath, D. T. Bowron, and A. K. Soper, J. Chem. Phys. 118, 273 (2003).

${ }^{10}$ G. Giraud, C. M. Gordon, I. R. Dunkin, and K. Wynne, J. Chem. Phys. 119, 464 (2003)

${ }^{11}$ J.-F. Huang, P-Y. Chen, I-W. Sun, and S. P. Wang, Inorg. Chim. Acta 7, 320 (2001).

${ }^{12}$ M. L. T. Asaki, A. Redondo, T. A. Zawodzinski, and A. J. Taylor, J. Chem. Phys. 116, 10377 (2002).

${ }^{13}$ (a) C. J. Margulis, H. A. Stern, and B. J. Berne, J. Phys. Chem. B 106, 12017 (2002); (b) T. I. Morrow and E. J. Maginn, ibid. 106, 12807 (2002); (c) C. G. Hanke, S. L. Price, and R. M. Lynden-Bell, Mol. Phys. 99, 801 (2001).

${ }^{14}$ F. Guillaume, G. Coddens, A. J. Dianoux, W. Petry, M. Rey-Lafion, and C. Sourisseau, Mol. Phys. 67, 665 (1989).

${ }^{15}$ K. F. Bradley, S.-H. Chen, and T. O. Brun, J. Chem. Phys. 95, 5273 (1991)

${ }^{16}$ J. J. Ullo and S. Yip, J. Chem. Phys. 85, 4056 (1986).

${ }^{17}$ J. G. Huddleston, H. D. Willauer, R. P. Swatlowski, A. E. Visser, and R. D. Rogers, Chem. Commun. (Cambridge) 1998, 1765.

${ }^{18}$ (a) C. M. Gordon, in Ionic Liquids in Synthesis, edited by P. Wasserscheid and T. Welton (Wiley-VCH, Weinheim, 2003), pp. 7-21; (b) L. Cammarata, S. Kazarian, P. Salter, and T. Welton, Phys. Chem. Chem. Phys. 3, $5192(2001)$

${ }^{19}$ (a) J. Dupont, C. S. Consorti, and J. Spencer, J. Braz. Chem. Soc. 11, 337 (2000); (b) J. G. Huddleston, A. E. Visser, W. M. Reichart, H. D. Willauer, G. A. Broker, and R. D. Rogers, Green Chem. 3, 156 (2001). 
${ }^{20}$ S. Hayashi, R. Ozawa, and H. Hamaguchi, Chem. Lett. 32, 498 (2003); but see also the recent S. Saha, S. Hayashi, A. Kobayashi, and H. Hamaguchi, ibid. 32, 740 (2003) and J. D. Holbrey, W. M. Reichert, M. Nieuwenhuyzen, S. Johnston, K. R. Seddon, and R. D. Rogers, Chem. Commun. (Cambridge) 2003, 1636.

${ }^{21}$ P. Wasserscheid, C. M. Gordon, C. Hilgers, M. J. Muldoon, and I. R. Dunkin, Chem. Commun. (Cambridge) 2001, 1186.

${ }^{22}$ J. Mesot, S. Janssen, L. Holitzner, and R. Hempelmann, J. Neutron Res. 3, 293 (1996).

${ }^{23}$ W. S. Howells, A fast Fourier transform program for the deconvolution of IN10 data, RL-81-039, Rutherford Appleton Laboratory, 1981.

${ }^{24}$ B. Frick and M. Gonzalez, Physica B 301, 8 (2001).

${ }^{25}$ B. Frick and L. J. Fetters, Macromolecules 27, 974 (1994).

${ }^{26}$ See, e.g., G. Zaccai, Science 288, 1604 (2000).

${ }^{27}$ G. Williams and D. C. Watts, Trans. Faraday Soc. 66, 80 (1970).

${ }^{28}$ See, e.g., K. L. Ngai, J. Non-Cryst. Solids 275, 7 (2000).
${ }^{29}$ A. Arbe, J. Colmenero, M. Monkenbusch, and D. Richter, Phys. Rev. Lett. 81, 530 (1998).

${ }^{30}$ See, e.g., the recent reports: A. Arbe, J. Colmenero, F. Alvarez, M. Monkenbusch, D. Richter, B. Farago, and B. Frick, Phys. Rev. E 67, 051802 (2003); J. Colmenero, F. Alvarez, and A. Arbe, ibid. 65, 041804 (2002); B. Farago, A. Arbe, J. Colmenero, R. Faust, U. Buchenau, and D. Richter, ibid. 65, 051803 (2002).

${ }^{31}$ A. Triolo, R. E. Lechner, A. Desmedt, M. T. F. Telling, and V. Arrighi, Macromolecules 35, 7039 (2002).

${ }^{32}$ See, e.g., A. Chahid, A. Alegria, and J. Colmenero, Macromolecules 27, 3282 (1994).

${ }^{33}$ V. Arrighi, P. F. Holmes, S. Gagliardi, I. J. McEwen, and M. T. F. Telling, Appl. Phys. A: Mater. Sci. Process. A74, S466 (2002).

${ }^{34}$ P. Gallo, F. Sciortino, P. Tartaglia, and S. H. Chen, Phys. Rev. Lett. 76, 2730 (1996).

${ }^{35} \mathrm{~A}$. Triolo et al. (unpublished).

${ }^{36} \mathrm{C}$. M. Gordon et al. (unpublished results). 